\section{Down's Syndrome and Human Behaviour}

GERMAN ${ }^{1}$ has proposed that Down's syndrome may be the result of delayed fertilization consequent to spcradic or decreased frequency of coitus. It has also been suggested ${ }^{1}$ that this hypothesis could be tested by comparing the incidence of this syndrome in similar groups differing only in the frequency of coitus and also by studying the incidence of the syndrome in the offspring of recently married older mothers and of unmarried mothers.

Many of these data would be difficult to obtain for obvious technical reasons; furthermore, the difference in frequency of coitus between young and old parental age groups is so small in absolute numbers ${ }^{2}$ that a rolatively large proportional difference between two compared groups might be difficult to detect.

I wish to draw attention to another behavioural factor which, if the forogoing hypothesis is correct, would actively promote the formation of zygotes with this type of chromosome abnormality. I refer to the use of the so called "safe period" as a means of contraception; this involves the avoidance of intercourse at the estimated time of ovulation and for several days before and after. Clearly failures of this method of contraception would be specifically associated with irregular ovulation and/or late fertilization.

Assuming the hypothesis, this factor would figure prominently in the production of Down's syndrome by younger mothers. The use of this contraceptive technique also provides a relatively easy method of testing the hypothesis, namely, the examination of data pertaining to the use of the "safe period" among parents of cases of Down's syndrome and comparable control groups. Groups such as those studied by Sigler and his colleagues ${ }^{3,4}$ would be ideally suited.

Because this contraceptive method is, as yet, the only technique approved officially by the Roman Catholic Church, it would be expected that parents of infants with Down's syndrome would be more frequently Roman Catholic. Sigler and colleagues ${ }^{4}$ found 50.9 per cent of 214 mothers of such cases to be Catholic, as compared with 43.3 per cent of 215 carefully matched controls. These differences do not reach the generally accepted levels of statistical significance, but the trend is in the expected direction. In the same study a much higher frequency of multiple marriages (before the birth of the index child) was noted among the mothers of the group with Down's syndrome. This finding has remained unexplained and although the difference was statistically significant $(0.01>P>0.001)$ the authors suggested that the significance might be more apparent than real and could be a chance association in a study where many factors were being evaluated. This seems unlikely, however, because multiple marriages associated with previous widowhood were equally common in both groups. Search for a further explanation therefore seems warranted. It also follows that attention should be directed to causes of divorce. A Philadelphia study ${ }^{5}$ of the causes of divorce found sexual maladjustment to be one of the four major complaints; either the husband declaring the wife to be little interested in sex relations or the wife claiming the husband to be over-demanding in terms of the frequency of coitus or of "abnormal" relations. In addition, Kinsev et $a l .{ }^{2}$ have hinted that women who are subsequently divorced evinced below average interest in coitus during their marriage. Under the proposed hypothesis it is at least possible that increased frequency of divorces amongst mothers of children with Down's syndrome may reflect a relatively low frequency of coitus among women who are subsequently divorced.

Penrose and Berg ${ }^{6}$ have recently published data on the duration of marriage among mothers of cases of Down's syndrome and a control group. Though they interpreted these data as not supporting German's hypothesis, the figures do show that the difference in duration of marriage among the oldest group of mothers was in the direction predicted by the hypothesis, although the "controls" were not drawn from the general population and the size of the oldest control group was small.

Increased maternal age also appears to be associated with other chromosome abnormalitios in man ${ }^{7-9}$ (Turner's syndrome being a notable exception ${ }^{10}$ ) and there are indications that these abnormalities, show some familial aggregation ${ }^{11}$. In view of these findings, and of the nonspecific nature of the chromosome abnormalities described in animals as a result of delayed fertilization ${ }^{12-14}$, it may be postulated that many chromosome abnormalities in man are associated with delayed fertilization and the use of the "safe period". This extension of the hypothesis could also be tested directly. Chromosome abnormalities are a relatively common cause of spontaneous abortions ${ }^{15,16}$ and if contraceptive and coital histories are obtained from women who have spontaneously aborted these data could be compared for those where chromosome abnormalities were detected in the foetal tissues as opposed to the others.

The extended hypothesis also gives rise to the prediction that the frequency of spontaneous abortions should be greater with increasing maternal age, and that a significant number of abortions should be associated with fertilization occurring late in the menstrual cycle. The results of earlier studies ${ }^{17,18}$ fulfil these predictions. The fact that some investigators ${ }^{4}$ have not shown a significantly in. creased frequency of abortions among mothers of cases of Down's syndrome may be due to the fact that only abnormalities due to chromosome anomalies would have a higher incidence in this group.

\section{J. Philip Welch}

Department of Paediatrics, Faculty of Medicine,

Dalhousie University,

Halifax, Nova Scotia.

Received May 12, 1968.

${ }^{1}$ German, J., Nature, 217, 516 (1968).

${ }^{2}$ Kinsey, A. C., Pomeroy, W. B., Martin, C. E., and Gebhard, P. H., Sexual Behaviour in the Human Female, 348, Table 93. 536 (W. B. Saunders Co., New York, 1953).

${ }^{3}$ Sigler, A. T., Lilienfeld, A. M., Cohen, B. H., and Westlake, J. E., $J$. Pediatrics, 67, 631 (1965).

${ }^{4}$ Sigler, A. T., Cohen, B. H., Lilienfeld, A. M., Westlake, J. E., and Hetznecker, W. H., J. Pediatrics, 70, B08 (1967)

Kephart, W. M., Marriage and Family Living, 16, 241 (1954).

- Penrose, L. S., and Berg, J. M., Nature, 218, 300 (1968).

7 Smith, D. W., Amer. J. Obstet. Gyne., 90, 1055 (1964).

${ }^{8}$ Welch, J. P., Lancet, i, 1229 (1967).

'Lenz, W., Pfeiffer, R. A., and Tünte, W., Dtsch. Med. Wschr., 91, 126? (1966).

${ }^{10}$ Boyer, S. H., Ferguson Smith, M. A., and Grumbach, M. M., Ann. Hum. Genet., 25, 215 (1961).

Hecht, F., Bryant, J. S., Gruber, D., and Townes, P. L., New Enff. J. Med., 27, 1081 (1964)

${ }^{12}$ Witschi, E., and Laguens, R., Develop. Biol., 7, 605 (1963).

13 Butcher, R. L, and Fugo, N. W., Fert. Ster. 18, 297 (1967).

${ }^{14}$ Austin, C. R., Nature, 213, 1018 (1967).

${ }^{15}$ Carr, D. H., Obstet. and Gynec., 26, 308 (1965).

16 Inhorn, S. L., Adv. Teratology, 37 (Academic Press, New York, 1967).

7 Iffy, L., Proc, Roy, Soc. Med, 56, 1098 (1963).

${ }_{18}$ Warburton, D., and Fraser, F. C., Amer. J. Hum. Genet., 16, 1 (1964).

\section{Methysergide in Mania}

I нAVE proposed a comprehensive theory of cerebral amine function which links biochemical, physiological, psychological and clinical phenomena ${ }^{1,2}$. One hypothesis derived from this general theory is that methysergide should benefit patients with mania. The results of trials in the first five patients are sufficiently notable for a preliminary account to be given.

All five patients had marked or severe signs of classical mania $^{3,4}$ without other complicating psychiatric or physical illness. There were two males and three females and their ages varied between 18 and 50. Three patients had previously been treated with chlorpromazine; two 